\title{
The Metal Surface Layer Mechanical Condition Transformation in Machining Processes
}

\author{
Valeriy Blumenstein ${ }^{1}$, and Maxim Makhalov ${ }^{1}$ \\ ${ }^{1}$ T.F. Gorbachev Kuzbass State Technical University, Mechanical engineering Technology \\ Department, 650000 Kemerovo, 28 Vesennya st., the Russian Federation
}

\begin{abstract}
The metal surface layer mechanical condition transformation at the product life cycle stages key provisions are presented. The described approach feature is the hardened body effect consideration: the metal mechanical properties changing during it displacement through the deformation zone space. On the basis of the developed for surface plastic deformation process hardened elastic-plastic body model, the cumulative shear strain level, plasticity reserve exhaustion level and residual stress tensor components calculations are performed. It is established that the greatest residual compressive stresses are characteristic for the axial component, and the extremum can be located both on the workpiece surface and at some distance from it. The metal hardening influence on the residual stresses distribution is revealed. On the axial (largest) component example shown that the difference between the maximum values is almost $30 \%$. The obtained result corresponds to the idea that the hardened metal having an increased yield strength allows a larger residual stresses presence.
\end{abstract}

\section{Introduction}

It is known that the metal mechanical condition largely determines product operational life, with this at the manufacture stages, including the machining stages, the metal experiences significant changes.

Currently, the surface layer (SL) predominant role in ensuring the machine parts durability is generally recognized [1-13]. Especially the part surface layer, which forming throughout the manufacture technological process, receives the greatest loading during operation.

The authors developed the scientific view about the surface layer mechanical condition transformation in machining processes and operation on the basis of cumulative shear strain level and plasticity reserve exhaustion level estimation and residual stress transformation in the deformation zones (DZ) under the stress-strain condition changing and with consideration of the metal properties transformation in DZ space (the hardened body effect) [14-15].

In General, the proposed approach provisions can be represented by the SL formation scheme (fig. 1). The life cycle is represented by cutting, surface plastic deformation (SPD) and fatigue loading stages. Fatigue stage includes the cyclic durability and cyclic crack resistance stages. 


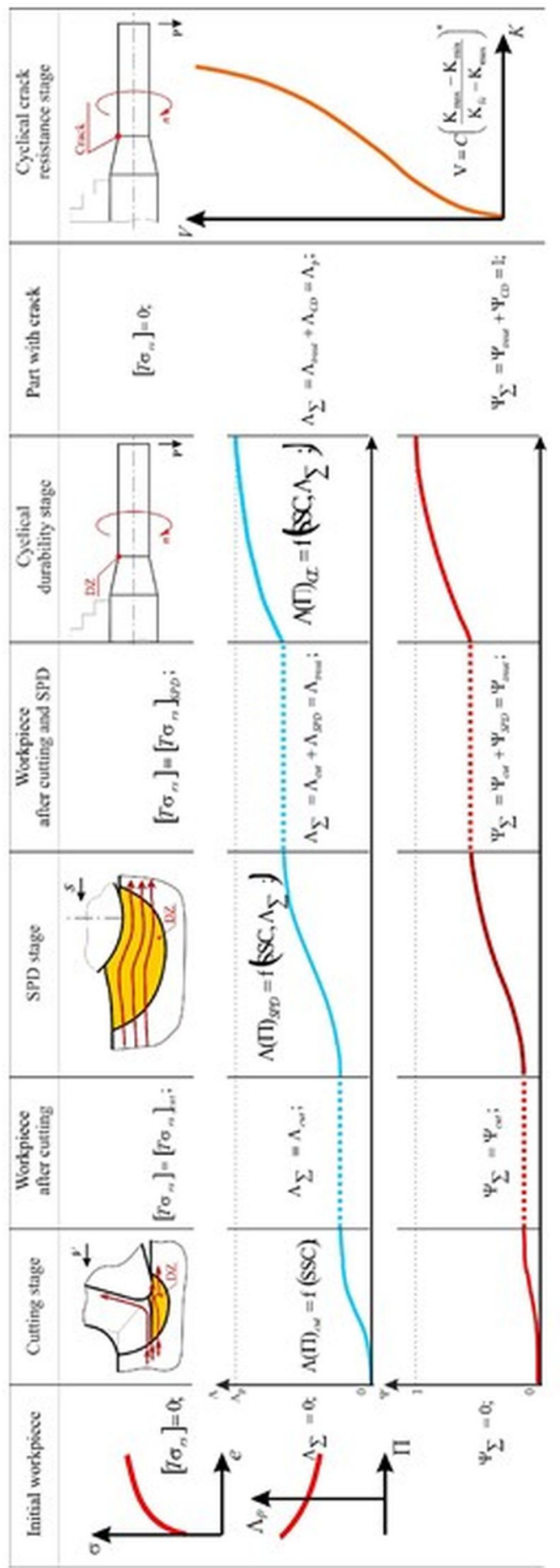

Fig.1. Surface layer metal properties transformation scheme at the product life cycle stages (SSCstress-strain condition) 
The hardening metal flow curve $\sigma=f(\varepsilon)$, ultimate plasticity $\Lambda_{p}=f(\Pi)$ and cyclic crack resistance $V=f(K)$ diagrams are used as initial conditions.

The main provisions of the proposed approach are:

- the SL mechanical condition transformation in the machining stages and subsequent operational loading are considered as a uniform continuous process of strain accumulation, plasticity reserve exhaustion and RS transformation in metal surface layer. Along with the traditional quality parameters, this process is described accordingly such parameter as: the cumulative shear strain level (CSSL) $\Lambda$, the plasticity reserve exhaustion level (PREL) $\Psi$ and residual stresses (RS) components tensor $\left[T \sigma_{r s}\right]$;

- the machining stages SL condition transformation occurs in the plastic deformation zone (DZ), appearing in the tool with the workpiece SL contact area;

- the mechanical condition formation occurs under the metal mechanical properties changing due to plastic deformation: loading history affects to the processes flow at every trajectory point inside DZ;

- at each machining or operation stage, the 1st kind RS formation occurs under the plastic strain influence, accumulated by the metal at the current time, and RS inherited from the previous stages;

- final mechanical condition and RS manifest themselves during the operation, transforming in each operating loading cycle; durability defines entire load prehistory.

- in the operational fatigue loading, the strain accumulation and plasticity reserve exhaustion processes continues, flowing under continuous residual stresses relaxation;

- strain free material corresponds to the value $\Psi=0$. With the strain limit accumulation $\left(\Lambda=\Lambda_{p}\right)$, there is a complete plasticity reserve exhaustion occurs $(\Psi=1)$. This condition corresponds to the first kind residual stress tensor relaxation to negligible values $\left(\left[T \sigma_{o c m}\right] \approx 0\right)$ and the fatigue crack appearance;

- a strain limit accumulation, a complete plasticity reserve exhaustion and fatigue crack nucleation occurs at a probable failure point, which may be located both on the surface and at some distance from it;

- further crack development is described in the cyclic crack resistance diagram categories in the "stress intensity factor $\mathrm{K}$ - fatigue crack growth rate $\mathrm{V}$ " coordinates.

According to taken assumptions, as the workpiece SL loading and unloading, accompanied by strain accumulation and plasticity reserve exhaustion, which occur in, stress condition scheme index changing conditions $\Pi$ :

$$
\Pi=\frac{\sigma}{T},
$$

where $\sigma$ - is the hydrostatic pressure (defined through the stress tensor first invariant)

$$
\sigma=\frac{I_{1}\left(T_{\sigma}\right)}{3}=\frac{\left(\sigma_{x x}+\sigma_{y y}+\sigma_{z z}\right)}{3}
$$

$\mathrm{T}$ - the shear stresses intensity (defined through the stress deviator second invariant) 


$$
\begin{aligned}
& T=\sqrt{\left|I_{2}\left(D_{\sigma}\right)\right|}= \\
& =\frac{1}{\sqrt{6}} \sqrt{\left(\sigma_{x x}-\sigma_{y y}\right)^{2}+\left(\sigma_{z z}-\sigma_{x x}\right)^{2}+\left(\sigma_{y y}-\sigma_{z z}\right)^{2}+6\left(\sigma_{x y}^{2}+\sigma_{y z}^{2}+\sigma_{z x}^{2}\right)}
\end{aligned}
$$

The cumulative shear strain level $\Lambda$ characterizes the strain condition and is defined as:

$$
\Lambda=\int_{0}^{t} H(\tau) d \tau,
$$

where $\mathrm{H}-$ is the shear strains velocity intensity (defined through the strain velocity deviator second invariant)

$$
\begin{aligned}
& H=2 \sqrt{\left|I_{2}\left(D_{\xi}\right)\right|}= \\
& =\sqrt{\left(\left(\xi_{x x}-\xi_{y y}\right)^{2}+\left(\xi_{y y}-\xi_{z z}\right)^{2}+\left(\xi_{z z}-\xi_{x x}\right)^{2}\right) \frac{2}{3}+4\left(\xi_{x y}^{2}+\xi_{y z}^{2}+\xi_{z x}^{2}\right)} .
\end{aligned}
$$

Stress condition scheme index values defines each loading or unloading step maximum cumulative shear strain level $\Lambda_{\mathrm{p}}$ at which the metal fatigue crack nucleation will occur:

$$
\Lambda_{p}=f(\Pi) .
$$

The plasticity reserve exhaustion level (PREL) $\Psi$ is a complex parameter, that describes as stress and strain material condition. According to the Kolmogorov linear model the plasticity reserve exhaustion level is defined as:

$$
\Psi=\int_{0}^{t} \frac{\Lambda}{\Lambda_{p}} d \tau .
$$

The first kind residual stresses values and distribution character over the parts cross section is one of the key metal mechanical condition parameters, largely determining the products operational life. According to some researchers, when an initial crack or other stress concentrator appears on the part surface, the surface layer compressing RS role in the fatigue life becomes primary and can give a several times increase in durability.

According to the unloading theorem, the residual stress tensor is the difference in stresses arising in a real elastoplastic body when a load $\left[T \sigma_{\text {def }}\right]$ is applied and the stresses that would appear in a perfectly elastic body under identical loading $\left[T \sigma_{\text {elast }}\right]$ summed with the elastic unloading stresses of the unfastening of the workpiece $\left[T \sigma_{\text {det }}\right]$ and the elastic thermal stresses of the unloading $\left[T \sigma_{t^{0}}\right][16]$ :

$$
\left[T \sigma_{\text {res }}\right]=\left[T \sigma_{\text {def }}\right]-\left[T \sigma_{\text {elast }}\right]+\left[T \sigma_{\text {det }}\right]+\left[T \sigma_{t^{0}}\right]
$$

SPD hardening treatment significantly increases the fatigue resistance before and after the fatigue crack appearance. It's known that for a low carbon steels content the fatigue strength increasing effect is due to an surface hardening increase, whereas for solid steels, 
it's explained by a great extent compressive residual stresses appearance, that inhibit a small cracks appearance and development.

In accordance with the above approach, the authors proposed and implemented for the SPD technology a hardened elastic-plastic body model. During the modeling the following initial conditions and assumptions was taken [14-15]:

1. Simulated isotropic material with the mechanical condition parameters corresponding to parameters of steel 45 (industry standard 1050-88, hardness 160-180 HV) in the delivery state.

2. The occuring strain had only mechanical nature due to the small metal heating in the loading process.

3. Flow curve linear approximation, taking into account the metal hardening, is used.

4. In view of the relatively low error value, to simplify the calculations, the Baushinger effect were not taken into account.

5. The workpiece releases unload elastic stresses tensors was adopted zero because a symmetrical cylindrical workpiece is being treated.

6. Possible boundary effects contribute to the simulation results a very small error, because workpiece model was large enough compared to the appearing deformation zone geometrical dimensions.

7. The roller tool was modeled as an absolutely rigid circle, because numerous researches have shown that when SPD processing, deforming tools are subjected to very little wear, and any significant rollers or balls deformations are not fixed.

Below are the residual stress components calculations results for the roller tool model with the following parameters: profile radius $R_{p r}=5 \mathrm{~mm}$, specified tension $h_{s}=0.05 \mathrm{~mm}$, feed $\mathrm{S}=0.1 \mathrm{~mm} /$ rot.

The largest compressive stress values, reaching up to - $600 \mathrm{MPa}$, has an axial component $\sigma_{\mathrm{x}}$, and for the considered processing routine, the compressive stress extremum located is located on the workpiece surface. The compressive stress distribution depth be approximately $3 \mathrm{~mm}$ at a significant stresses depth distribution about $7 \mathrm{~mm}$ (fig. 2) [16].

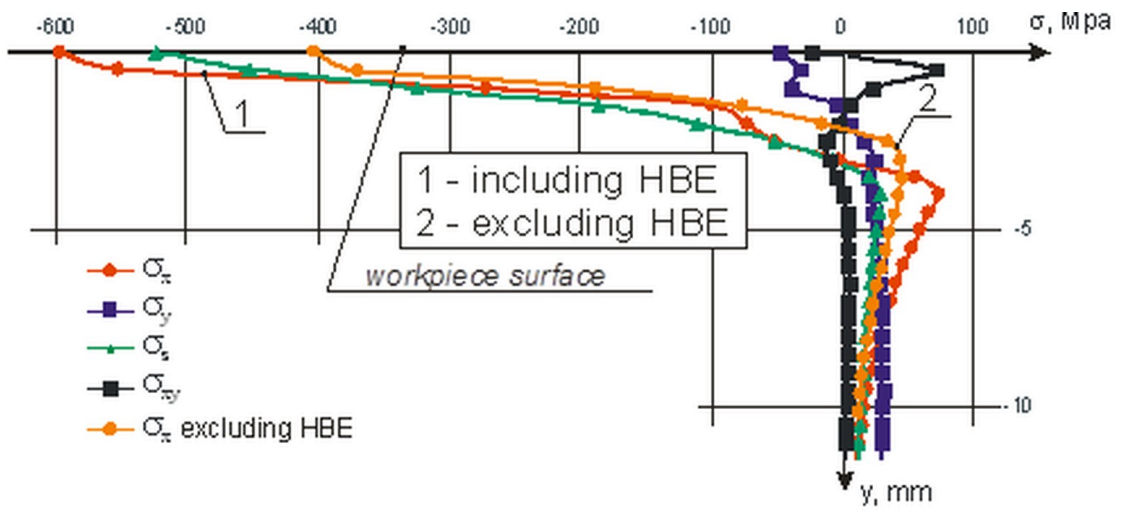

Fig. 2. The surface layer depth residual stress tensor components distribution. Axial residual stresses $\sigma_{\mathrm{x}}$ including (1) and excluding (2) the hardened body effect (HBE)

The tangential (circumferential) component $\sigma_{\mathrm{z}}$ in the chosen plane-strain formulation has a compressive stresses values comparable with the axial component (up to $-520 \mathrm{MPa}$ ). Maximum values also occur on the workpiece surface.

The radial component $\sigma_{\mathrm{y}}$ is characterized by much smaller values and, accordingly, smaller differences, both in the tensile and compressive values region at the same distribution depths. On the surface and in the nearby layer, the radial component is predominantly negative (up to $-50 \mathrm{MPa}$ ). 
This solution feature is to determine the tangent component $\sigma_{\mathrm{xy}}$, which distribution has complex character with a relatively small stress values on the surface and at some surface layer depth.

A comparative analysis with a similar model that does not consider the hardened body effect (HBE) showed that the model with HBE detects larger compressive stresses values. The maximum compressive RS values difference between the model without hardening relative to the model with HBE considering is almost $30 \%$. The obtained result corresponds to the idea that the hardened metal having an increased yield strength, wich allows a larger residual stresses presence.

The radial RS component in the model with $\mathrm{HBE}$ is also characterized by 2 times greater both compressive and tensile stresses values.

The circumferential (tangential) component in both models has mainly compressive values, the extremum located on the workpiece surface. As for the radial component, the model with hardening has 2 times greater stress values of both signs.

The feed plane tangent component for both cases has a similar distribution, but the model that consider the HBE has a slightly larger final values range.

\section{Conclusions}

1. A proposed approach feature is the TI phenomenon consideration not only at the previous loading (treatment or operation) stages, but at the current loading stage level, during metal particles displacement through the deformation zone space (the hardened body effect).

2. On the developed hardened elastic-plastic body model for the SPD process, the residual stress tensor components calculations are performed. It's established that the greatest residual compressive stresses are characteristic for the axial component, and the extremum can be located both on the workpiece surface and at some distance from it. The compressive RS distribution depth is about $3 \mathrm{~mm}$ at a significant stresses depth distribution about $7 \mathrm{~mm}$.

3. The metal hardening influence on the residual stresses distribution is revealed. On the axial (largest) component example shown that the difference between the maximum values is almost $30 \%$. The obtained result corresponds to the idea that the hardened metal having an increased yield strength allows a larger residual stresses presence.

\section{References}

1. Yuan Ma, Pingfa Feng, Jianfu Zhang, Zhijun Wu, Dingwen Yu Int J Adv Manuf Technol., 81, 1023 (2015)

2. Xiaoming Huang, Jie Sun, Jianfeng Li Int J Adv Manuf Technol., 77, 1035 (2015)

3. Jiann-Cherng Su, Keith A. Young, Kong Ma, Shesh Srivatsa, John B. Morehouse, Steven Y. Liang Int J Adv Manuf Technol., 65, 717 (2013)

4. Xia Ji, Xueping Zhang, Steven Y. Liang Int J Adv Manuf Technol., 70, 2159 (2014)

5. Joseph J. Martell, C. Richard Liu, Jing Shi Int J Adv Manuf Technol., 74, 1381 (2014)

6. Jianbin Chen, Qihong Fang, Liangchi Zhang Int J Adv Manuf Technol., 75, 615 (2014)

7. T. Altan Manufacturing Technology, 54, 237 (2017)

8. M. Sayahi, S. Sghaier, H. Belhadjsalah Int J Adv Manuf Technol., 67, 1665 (2013) 
9. J. J. Liou, T. I. El-Wardany International Journal of Manufacturing Engineering, 2014, 1 (2014)

10. A. Gopinath, A Lim, B. Nagarajan, CC Wong, R. Maiti, S. Castagne, IOP Conference Series: Materials Science and Engineering, 157 (2013)

11. Partchapol Sartkulvanich, T. Altan, F. Jasso, C. Rodriguez J. Manuf. Sci. Eng., 129, 705 (2007)

12. Xiaohui Jiang, Beizhi Li, Jianguo Yang, XiaoYan Zuo, Kang Li Int J Adv Manuf Technol., 66, 1439 (2013)

13. Xiaohui Jiang, Beizhi Li, Jianguo Yang, Xiao Yan Zuo Int J Adv Manuf Technol., 68, 175 (2013)

14. V.Yu. Blumenstein, O.A. Ostanin, M.S. Mahalov E3S Web Conferences, IIIrd International Innovative Mining Symposium, 41, 3 (2018)

15. M.S. Mahalov, V.Yu. Blumenstein, Metal Working and Material Science, 20, 6 (2018)

16. M.S. Mahalov, V.Yu. Blumenstein, Metal Working and Material Science, 21, 110 (2019) 